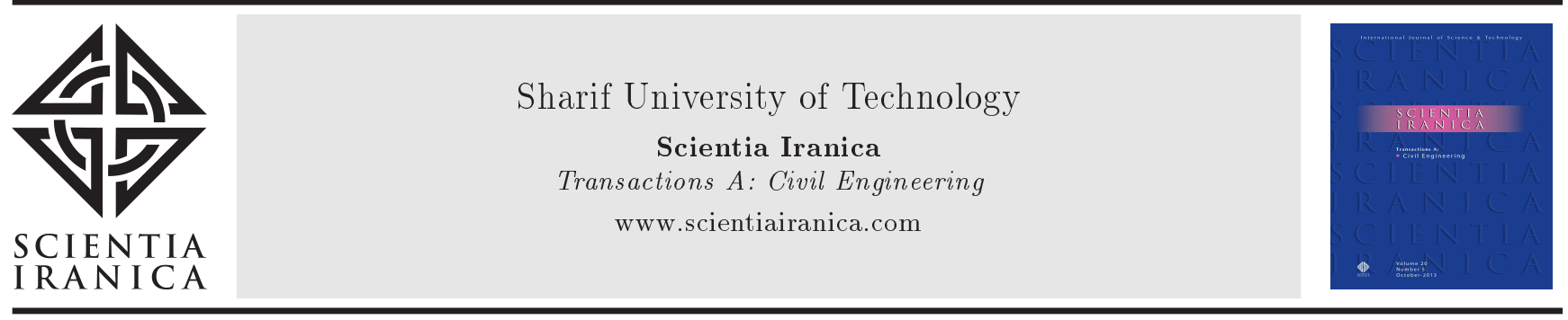

\title{
Dam break model with Eulerian equations using mapping technique
}

\author{
A. Lohrasbi* and M.D. Pirooz \\ School of Civil Engineering, College of Engineering, University of Tehran, Tehran, Iran.
}

Received 22 April 2015; received in revised form 25 July 2015; accepted 15 September 2015

\section{KEYWORDS}

Dam break;

Eulerian equations;

Free-surface;

Mapping technique.

\begin{abstract}
This paper deals with numerical modeling of water flow, which is generated by the break of a dam. The problem is solved by applying Eulerian equations with mapping technique over a horizontal bottom considering unsteady incompressible flow with free surface. The proposed model has been used to simulate a two-dimensional problem of the collapse of a water column inside a rectangular tank. A new mapping is developed to transform the governing equations from the physical domain to a computational domain. It is shown that the model predicts the changes of wave profile and the free surface elevation of water. In addition, the results are compared with experimental data.
\end{abstract}

(C) 2016 Sharif University of Technology. All rights reserved.

\section{Introduction}

When a dam is breached, catastrophic flooding occurs in the downstream channel. Dam break analyses are used to estimate the potential hazards associated with a structure failure. Zienkiewicz et al. [1] studied flows due to dam breaking by using the Lagrangian description. Pohle [2] and Stoker [3] presented a systematic procedure for the determination of the successive terms in these expansions. However, only the leadingorder terms were constructed and analyzed. In both Lagrangian and Eulerian descriptions, the expansions of the solution were in time power. However, such a solution was successfully derived in a relevant problem concerning a uniformly accelerating wave-maker by King and Needham [4]. There are numerous numerical and experimental studies on dam break flows. Relevant to this study is the paper by Stansby et al. [5] in which the initial stage of dam break flow, for dry and wet bed cases, is studied, experimentally, and it is observed that for the dry bed case, a horizontal jet

*. Corresponding author. Tel.: +982144258217 E-mail addresses: ar_lohrasbi@ut.ac.ir (A. Lohrasbi); mdolat@ut.ac.ir (M.D.Pirooz) forms at small times. They also performed a numerical analysis on the dam break problem. However, in order to avoid the singularity of the numerical solution at the intersection point, they had to pre-wet the bed in front of the dam by an artificial thin fluid layer. Several numerical studies, performed during the past few years, were based on the solution of Nonlinear Shallow Water Equations (NSWE) using different methods such as the finite-volume method, the finitedifference method [6-10]. Hunt [11] used a kinematic wave approximation to obtain a closed-form solution for a sloping channel and mentioned that his solution was valid for large times. The dam break problem was interpreted in the context of a liquid column collapsing under gravity. Penney and Thornhill [12] studied the collapse of a fluid column, which was surrounded by a lighter fluid. The analysis was performed in Eulerian variables for both small and moderate times. They derived the initial asymptotics of the solution for fluid columns of semi-cylindrical and hemispherical shapes, and showed that these asymptotics are not correctly close to the base, where the fluid velocity is much higher than that in the rest of the column. Korobkin and Yilmaz [13] studied the initial stages of the dam break flow in the framework of potential free 
surface flows. Dutykh and Mitsotakis [14] solved the classical dam break problem of validating the nonlinear shallow water equations solvers and tried to examine the validity of the mathematical model under NavierStokes simulations. Lohrasbi et al. [15] presented an approach to solve Naiver-Stokes equations based on mapping technique about dam break. They used Arbitrary Lagrangian Eulerian (ALE) for transforming physical domain to computational domain. However, their solution had some limitations on ratio of upstream water height to downstream water height and had some errors, such as shock waves, on free surface. In this article, new mapping is presented that shows accurate results.

In this paper, in addition to the extraction of approximation functions, correction of their coefficient is also carried out. This modification improves the accuracy of the model and gives appropriate results to the previous works.

\section{Formulation of the problem}

The unsteady plane problem of free surface flow is considered, which is generated when a vertical dam in front of a liquid region is suddenly removed. Then, the liquid flow and the shape of its free surface during the early stages of the process will be determined. Initial condition of the liquid is shown in Figure 1. The physical domain $\bar{V}$ surrounded by a piecewise smooth boundary $\bar{S}$ is shown in Figure 2. This two-dimensional domain is occupied by a non-viscous incompressible fluid with the specific mass of $\rho$. The problem under consideration is the unsteady motion of a surface wave under gravity. The governing equations are expressed by the unsteady Eulerian equation and the equation of continuity. The rectangular coordinates are denoted by $x$ and $y$ and the corresponding velocity components are denoted by $u$ and $v$. As a result, the equations of conservation of momentum and

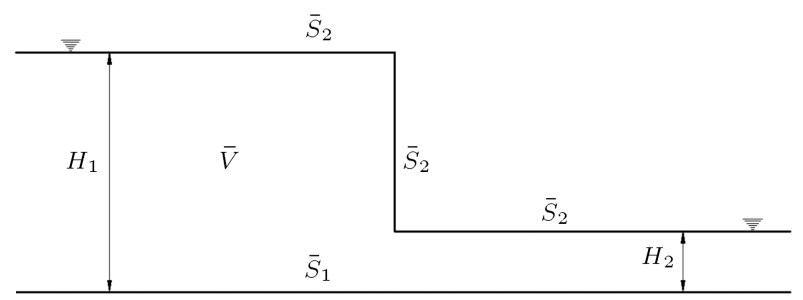

Figure 1. Initial condition of the liquid at time $t=0 \mathrm{sec}$.

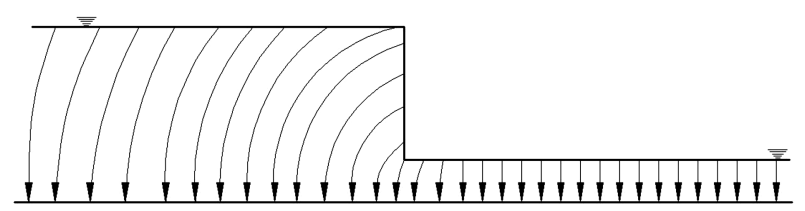

Figure 2. The computational grid is shown mapped back to the physical space. mass for incompressible Newtonian fluids are given as follows:

$$
\begin{gathered}
\left.\frac{\partial \bar{u}}{\partial \bar{t}}\right|_{\xi, \eta}+\left(\bar{u}-\bar{w}_{u}\right) \frac{\partial \bar{u}}{\partial \bar{x}}+\left(\bar{v}-\bar{w}_{v}\right) \frac{\partial \bar{u}}{\partial \bar{y}} \\
=-\frac{1}{\rho} \frac{\partial \bar{p}}{\partial \bar{x}} \\
\left.\frac{\partial \bar{v}}{\partial \bar{t}}\right|_{\xi, \eta}+\left(\bar{u}-\bar{w}_{u}\right) \frac{\partial \bar{v}}{\partial \bar{x}}+\left(\bar{v}-\bar{w}_{v}\right) \frac{\partial \bar{v}}{\partial \bar{y}} \\
=-\frac{1}{\rho} \frac{\partial \bar{p}}{\partial \bar{y}}-\bar{g} \\
\frac{\partial \bar{u}}{\partial \bar{x}}+\frac{\partial \bar{v}}{\partial \bar{y}}=0,
\end{gathered}
$$

where $w_{u}$ and $w_{v}$ are the mesh velocities in $x$ and $y$ directions. The boundary $\bar{S}$ consists of two types of boundaries: one is $\bar{S}_{1}$, on which velocity is given; the other is the free surface boundary $\bar{S}_{2}$, on which the surface force is specified. The boundary conditions are expressed as the followings:

$$
\begin{aligned}
& \bar{u}=\hat{\bar{u}} \text { on } \bar{S}_{1} \bar{p} \cdot n_{\bar{x}}=\hat{\bar{c}}_{x} \text { on } \bar{S}_{2}, \\
& \bar{v}=\hat{\bar{v}} \text { on } \bar{S}_{1} \bar{p} \cdot n_{\bar{y}}=\hat{\bar{c}}_{y} \text { on } \bar{S}_{2},
\end{aligned}
$$

where the superscript caret denotes a function which is given on the boundary and $n_{\bar{x}}$ and $n_{\bar{y}}$ symbolize the direction cosines of the outward normal to the boundary with respect to coordinates $x$ and $y$. Also, $\hat{\bar{c}}_{x}$ and $\hat{\bar{c}}_{y}$ are the constants of integration. The above equations can be rendered dimensionless by introducing the following variables:

$$
\begin{aligned}
& \bar{x}=x \bar{d}, \quad \bar{y}=y \bar{d}, \quad \bar{p}=p \overline{\rho g} \bar{d}, \\
& \bar{u}=u(\bar{g} \bar{d})^{1 / 2}, \quad \bar{v}=v(\bar{g} \bar{d})^{1 / 2}, \\
& \bar{t}=t\left(\frac{\bar{d}}{\bar{g}}\right)^{1 / 2} .
\end{aligned}
$$

Using these transformations, Eq. (1) can be modified as follows:

$$
\begin{gathered}
\frac{\partial u}{\partial t}+\left(u-w_{u}\right) \frac{\partial u}{\partial x}+\left(v-w_{v}\right) \frac{\partial u}{\partial y} \\
=-\frac{\partial p}{\partial x}, \\
\frac{\partial v}{\partial t}+\left(u-w_{u}\right) \frac{\partial v}{\partial x}+\left(v-w_{v}\right) \frac{\partial v}{\partial y} \\
=-\frac{\partial p}{\partial y}-1, \\
\frac{\partial u}{\partial x}+\frac{\partial v}{\partial y}=0 .
\end{gathered}
$$




\section{Free surface formulation}

The fluid surface equation is written as:

$$
\bar{F}=\bar{h}(x, t)+\bar{d}-\bar{y}=0 \text { on } \bar{S}_{2},
$$

where $h$ is the position of the free surface. The kinematic condition associated with the fluid free surface is defined as:

$$
\frac{D \bar{F}}{D \bar{t}}=0
$$

then:

$$
\frac{\partial \bar{F}}{\partial \bar{t}}+\left(\bar{u}-\bar{w}_{u}\right) \frac{\partial \bar{F}}{\partial \bar{x}}+\left(\bar{v}-\bar{w}_{v}\right) \frac{\partial \bar{F}}{\partial \bar{y}}=0
$$

With substituting into Eq. (5), the following is obtained:

$$
\begin{aligned}
\left(\frac{\partial \bar{h}}{\partial \bar{t}}-\frac{\partial \bar{y}}{\partial \bar{t}}\right) & +\left(\bar{u}-\bar{w}_{u}\right) \frac{\partial \bar{h}}{\partial \bar{x}} \\
& +\left(\bar{v}-\bar{w}_{v}\right)(-1)=0 .
\end{aligned}
$$

Utilizing the dimensionless form of the free surface kinematic equation written as:

$$
\begin{aligned}
\left(\frac{\partial h}{\partial t}-\frac{\partial y}{\partial t}\right) & +\left(u-w_{u}\right) \frac{\partial h}{\partial x} \\
& +\left(v-w_{v}\right)(-1)=0 .
\end{aligned}
$$

Also, Eq. (9) is simplified as:

$$
\frac{\partial h}{\partial t}+\left(u-w_{u}\right) \frac{\partial h}{\partial x}-v=0 .
$$

\section{Transformation of equations}

For the finite element method, such problem requires a complicated interpolation function on the local grid lines, which results in the local loss of accuracy in the computational solution. Such difficulties require a mapping or transformation from physical space to a generalized space. This transformation simplifies the problem. Lohrasbi et al. [15] presented this mapping that transforms the wave propagation model from the physical domain:

$$
\begin{aligned}
& x=\sum_{i=1}^{3}\left(\xi+h \alpha_{i}\right) F_{i}(\eta), \\
& y=\eta(1+h) .
\end{aligned}
$$

This transformation has limitation on the ratio of upstream to downstream water levels. So, in this research and for showing more and better results, the Lagrange interpolation formulations is used such as:

$$
\begin{aligned}
& x=\sum_{i=1}^{n}\left(\xi+h \alpha_{i}\right) \Pi_{i=0}^{n} \frac{\eta-\eta_{i}}{\eta_{k}-\eta_{i}} \quad(k=0,1,2, \ldots, n) \\
& y=\eta(1+h) .
\end{aligned}
$$

The $\alpha_{i}$ values are considered the fifth-order polynomial function of $\xi$ as follows:

$$
\begin{aligned}
\alpha_{i}= & \frac{b}{\varepsilon^{3} l^{5}(1-\varepsilon)^{3}}\left[2\left(\xi-\xi_{0}\right)^{5}(2 \varepsilon-1)+l\left(\xi-\xi_{0}\right)^{4}\right. \\
& \left(4-5 \varepsilon-5 \varepsilon^{2}\right)+2 l^{2}\left(\xi-\xi_{0}\right)^{3}\left(-1-\varepsilon+5 \varepsilon^{2}\right) \\
& \left.+l^{3} \varepsilon\left(\xi-\xi_{0}\right)^{2}(3-5 \varepsilon)\right] .
\end{aligned}
$$

Definitions of $b, \varepsilon, l$, and $\zeta_{0}$ are illustrated in Figure 3.

\section{Numerical example}

For dam break modelling with Navier-Stokes equations in this approach, the physical domain has been discreted to $\Delta x=\Delta y=0.25 \mathrm{~m}$ and 200 elements in horizontal and 4 elements in vertical directions in space and $\Delta t=0.01 \mathrm{sec}$ in time as shown in Figure 4. Free surfaces of dam at $t=0.0$ to $t=1.1 \mathrm{sec}$ are shown in Figure 5 and Figure 6 and surface profile in several times is shown in Figure 7 . For better

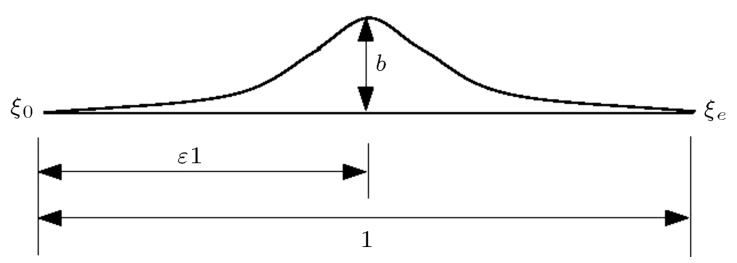

Figure 3. Definition of parameters in function $\alpha_{i}$.

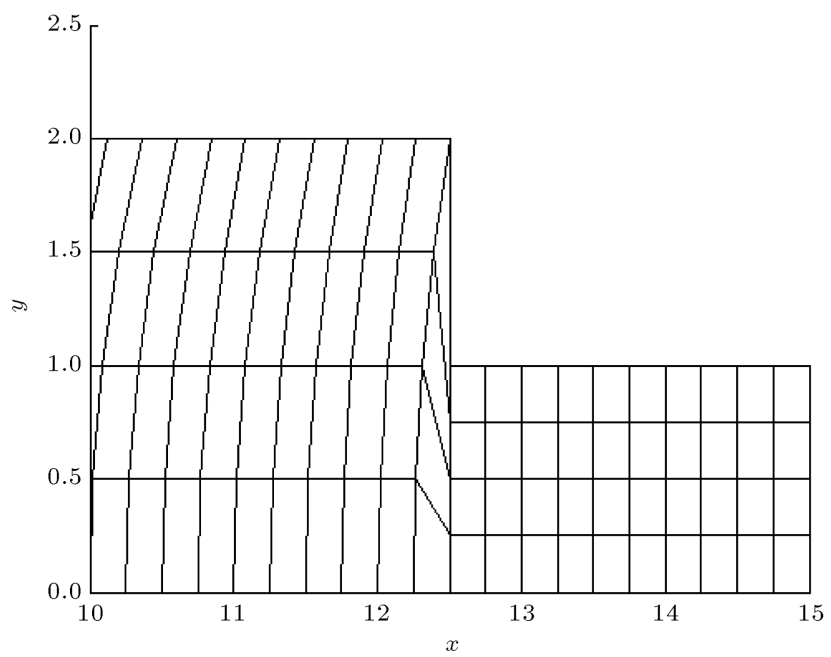

Figure 4. Physical domain meshing. 

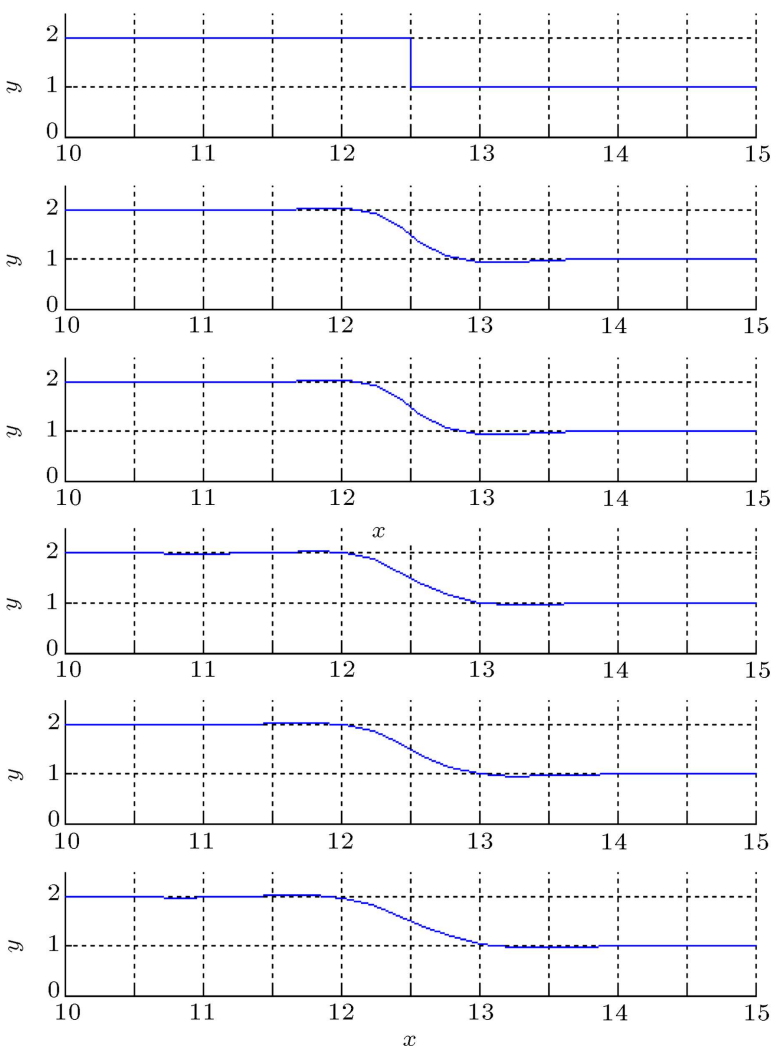

Figure 5. Free surface in dam break at time $=0.0,0.1$, $0.2,0.3,0.4,0.5 \mathrm{sec}$.
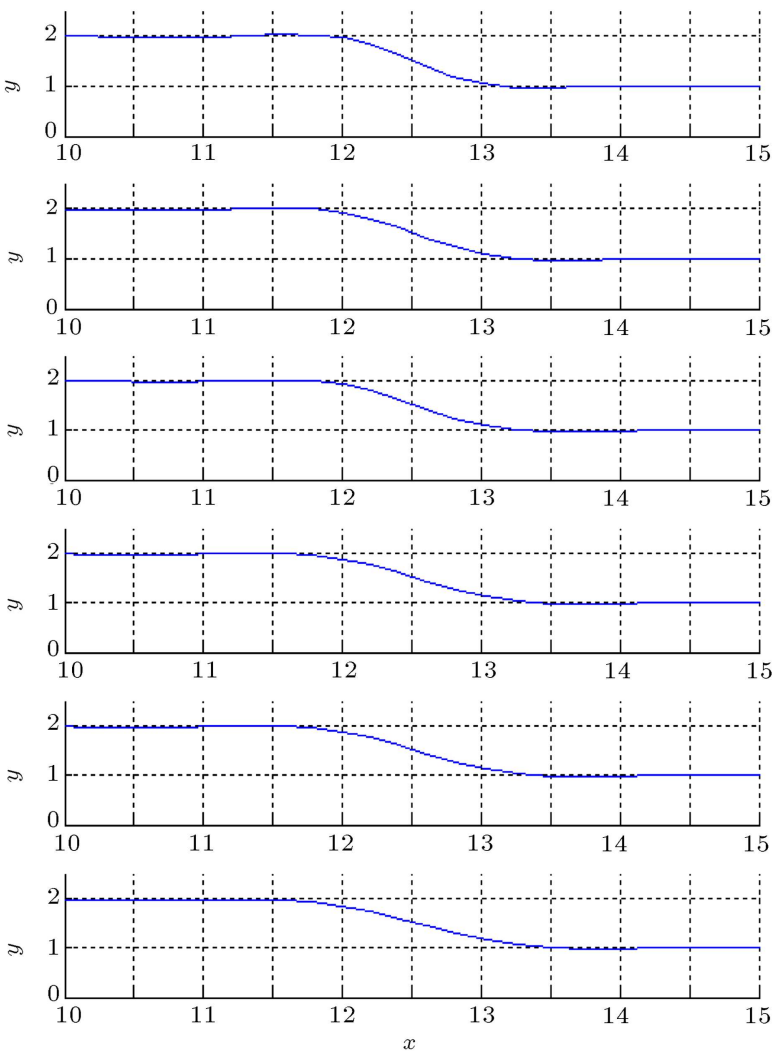

Figure 6. Free surface in dam break at time $=0.6,0.7$, $0.8,0.9,1.0,1.1 \mathrm{sec}$.
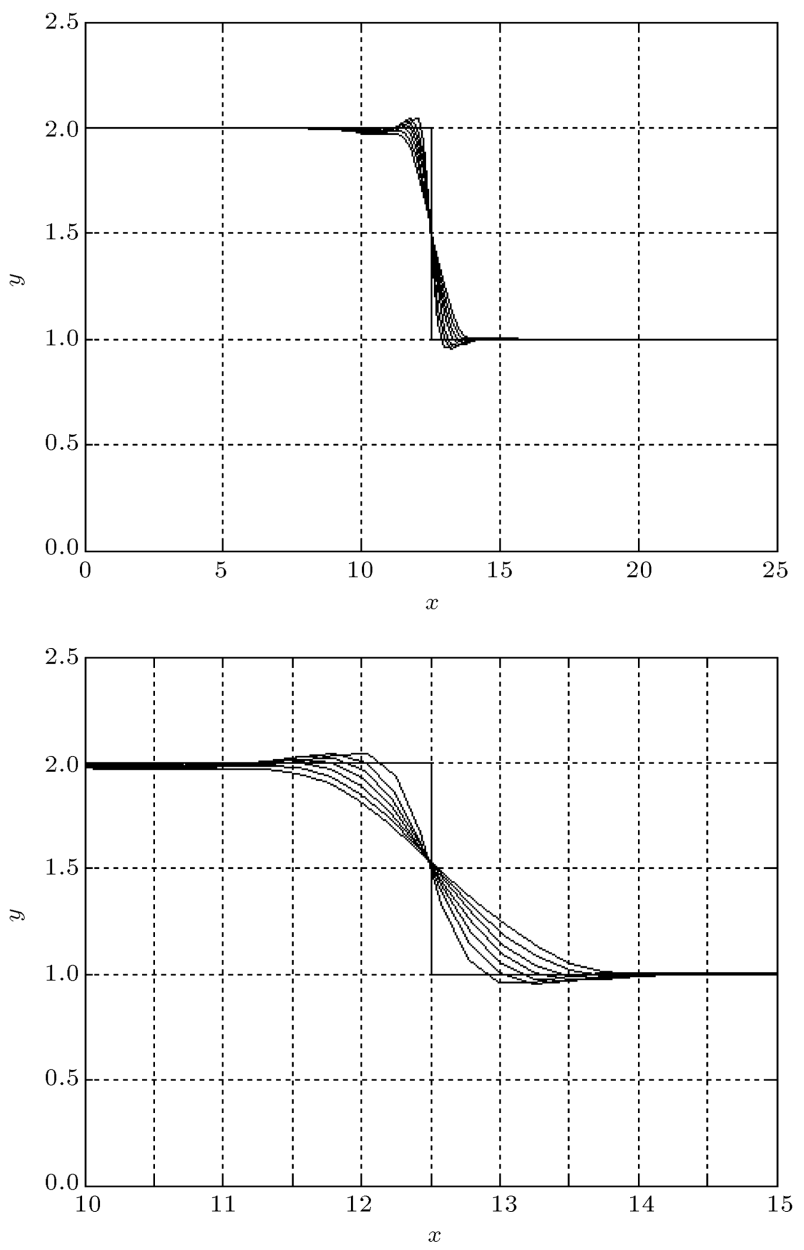

Figure 7. Free surface profile in several times.

demonstration of the results, variation of horizontal and vertical velocities is shown in Figures 8-10. The results of present numerical model have been compared with the results of Zienkiewicz [1] at $t=0.0\left(H_{1} / H_{2}=\right.$ 2.0) in Figure 11. Comparison between the current model and the numerical results of Zienkiewicz [1] shows acceptable results. This model has the capability of dam break model, such as $H_{1} / H_{2}=4.5$ that is illustrated in Figure 12. This ratio is the limitation of solution and has some errors, such as shock waves, on free surface.

\section{Conclusion}

The propagation and deformation of free surface in dam break over flat bathymetry is investigated. Here, a dam separating two stationary water levels is suddenly removed and the almost vertical waves progress into the two domains. This numerical approach is very suitable for solving Eulerian equation while maintaining the accuracy of calculations. The new mapping technique transforms the physical domain with the vertical wall to a simple rectangle computational domain. This transformation has no limitation on the ratio of up- 

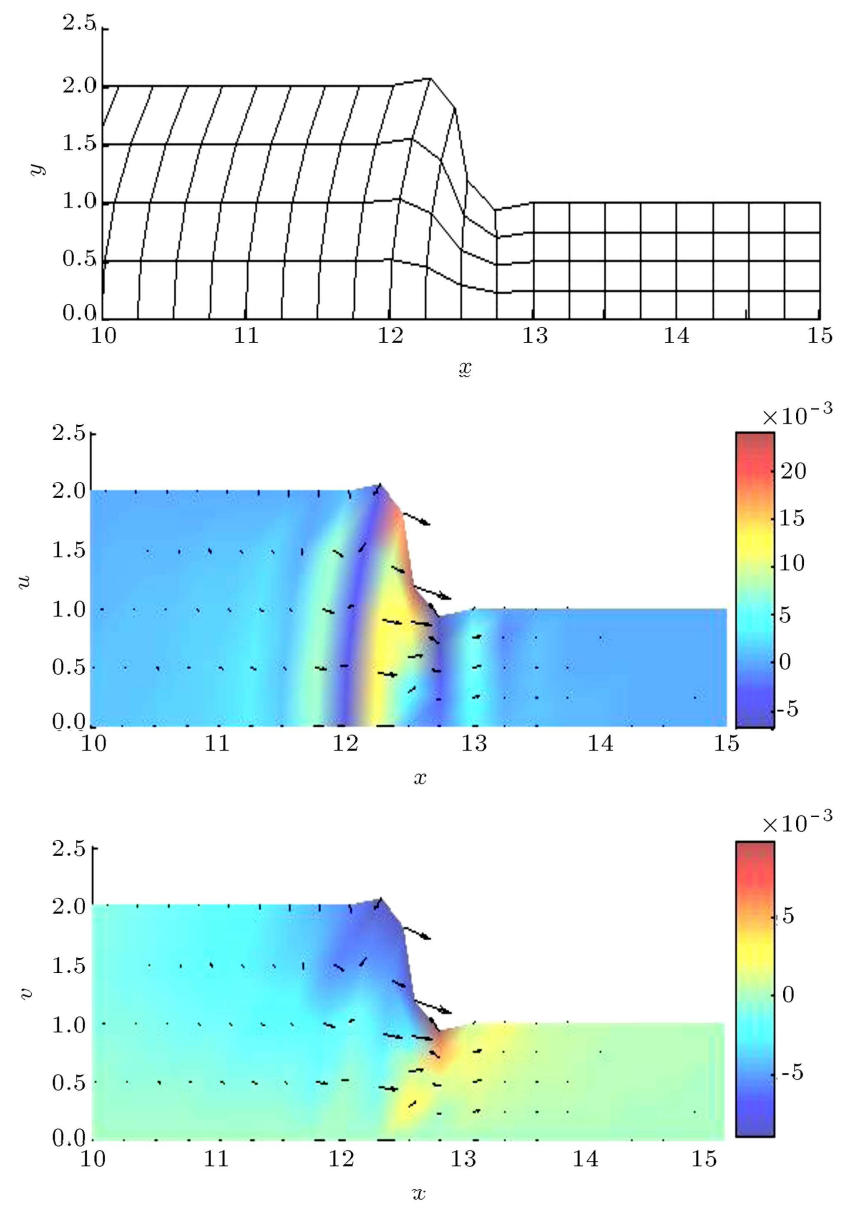

Figure 8. Free surface, horizontal and vertical velocities in domain in $t=0.1 \mathrm{sec}$.
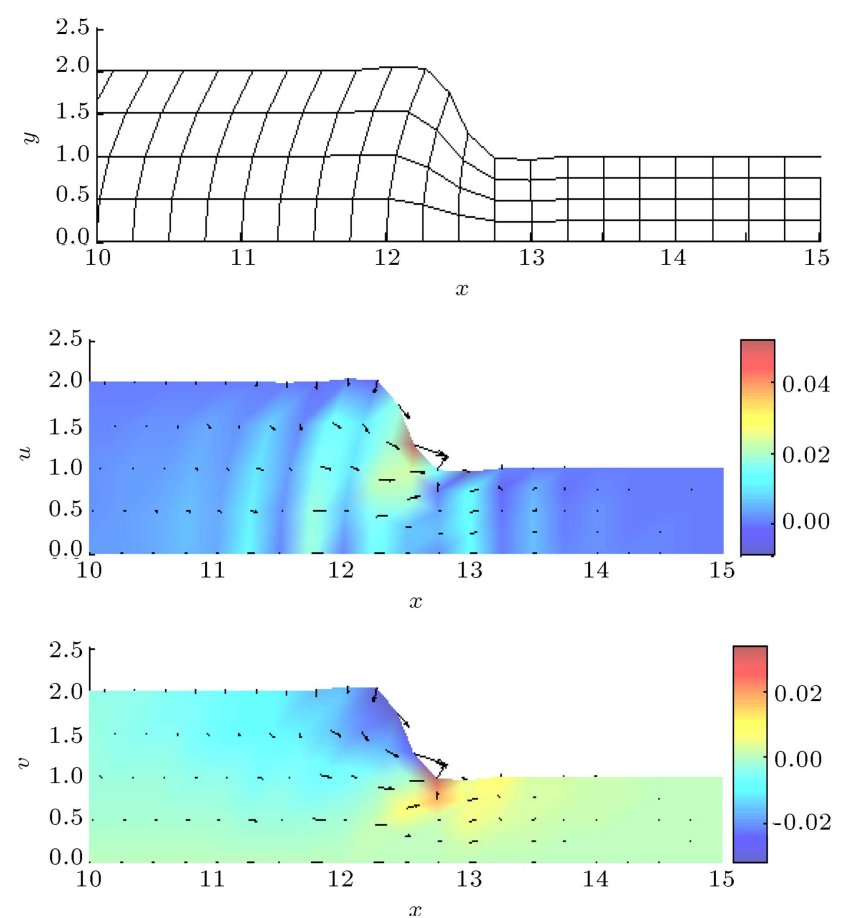

Figure 9. Free surface, horizontal and vertical velocities in domain in $t=0.6 \mathrm{sec}$.
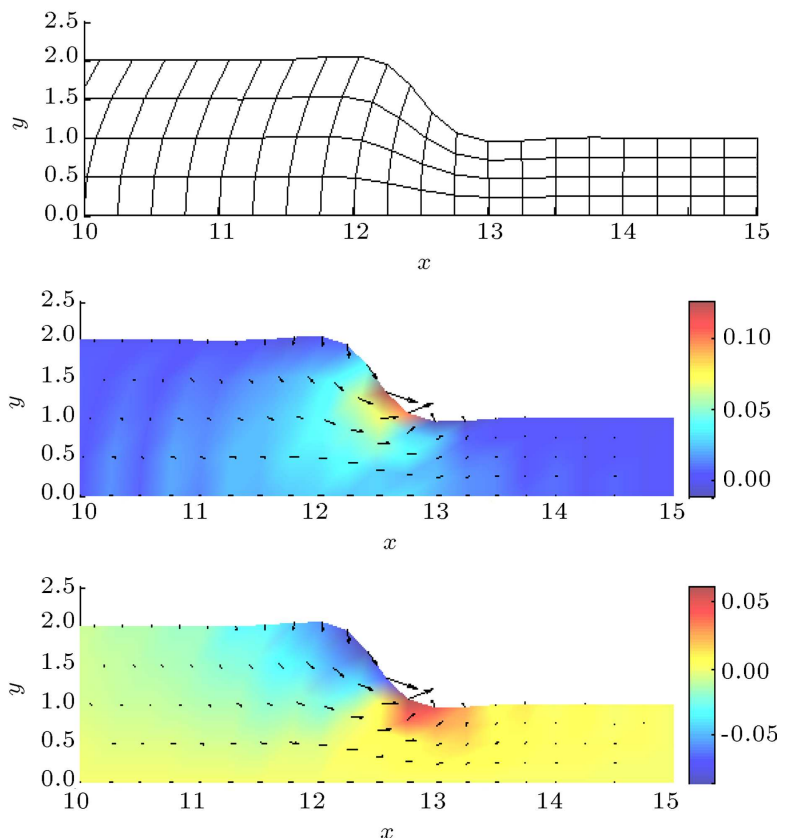

Figure 10. Free surface, horizontal and vertical velocities in domain in $t=1.1 \mathrm{sec}$.
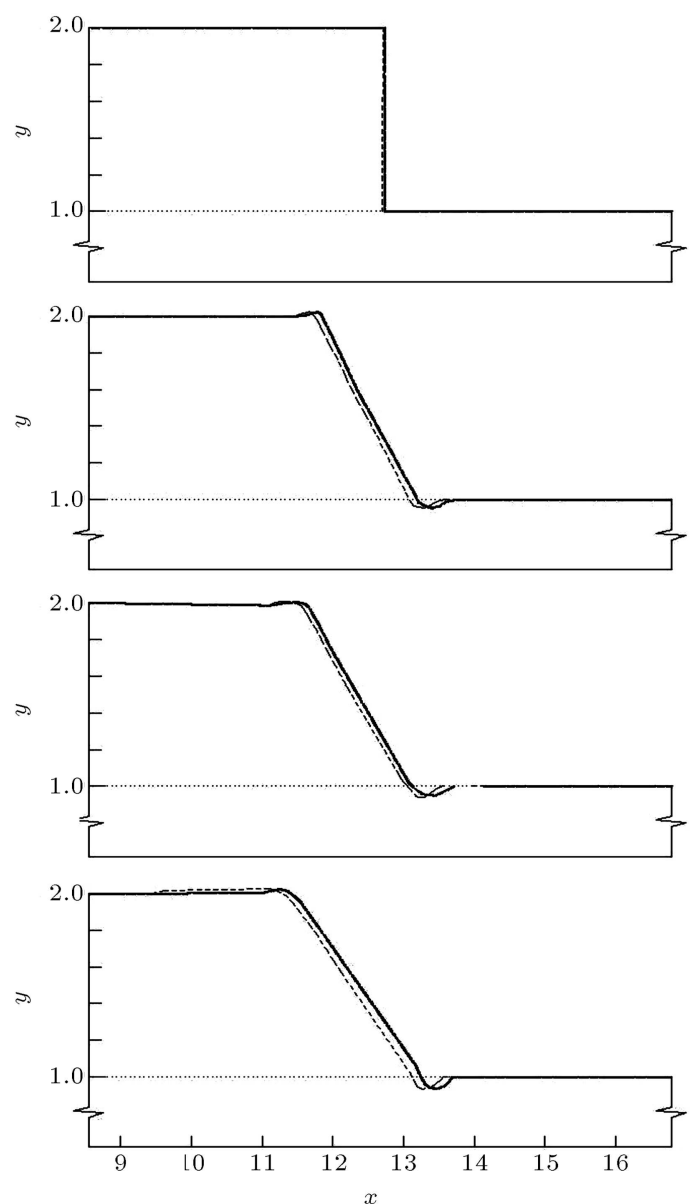

Figure 11. Comparison of the present model with Zienkiewicz numerical model with $H_{1} / H_{2}=2.0$ (full line for the current model and dashed line for Zienkiewicz results). 

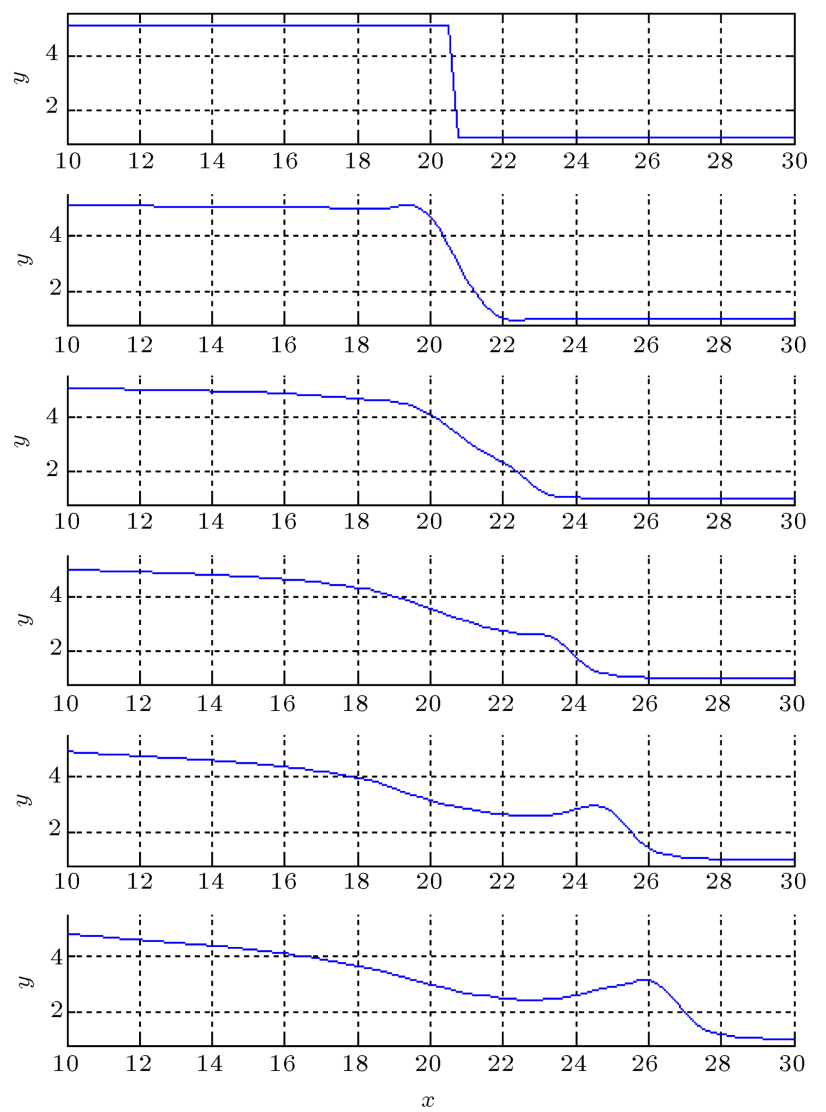

Figure 12. Dam break with the present numerical model $\left(H_{1} / H_{2}=4.5\right)$.

stream to downstream water levels unlike the previous research [15]. The model is validated by comparing numerical results with results obtained numerically. Overall, the conformity of the available data with the computations is well and in most cases, the numerical model gives excellent results. The method can be applied to other unsteady waves and it provides appropriate agreement with independent calculations of other research.

\section{References}

1. Zienkiewicz, R., Taylor, L. and Zhu, J.Z., The Finite Element Method: Its Basis and Fundamentals, 6th Edition, Elsevier, Oxford (2005).

2. Pohle, F. The Lagrangian equations of hydrodynamics: Solutions which are analytic functions of time, New York, University U.S.A. (1950).

3. Stoker, J., Water Waves: The Mathematical Theory with Applications, Pure and Applied Mathematics, IV, Interscience Publishers, Inc., New York, Interscience Publishers Ltd., London (1957).

4. King, A. and Needham, D. "The initial development of a jet caused by fluid, body and free surface interaction", J. Fluid Mech, 268, pp. 89-101 (1994).
5. Stansby, P.K., Chegini, A. and Barnes, T. "The initial stages of dam-break flow", J. Fluid Mech, 374, pp. 407-424 (1998).

6. Zoppou, C. and Roberts, S. "Explicit schemes for dambreak simulations", J Hydraul Eng., 129, pp. 11-34 (2003).

7. Zoppou, C. and Roberts, S. "Numerical solution of the two-dimensional unsteady dam break", Appl. Math. Fluids Res., 24, pp. 457-475 (2000).

8. Luigi, F. and Toro, E.F. "Experimental and numerical assessment of the shallow water model for two dimensional dambreak problems", J. Hydraul. Res., 33, pp. 843-864 (1995).

9. Glaister, P. "Solutions of a two dimensional dam break problem", Int. J. EngSci., 29, pp. 1357-1362 (1991).

10. Brufau, P. and Garcia-Navarro, P. "Two dimensional dam break flow simulation", Int. J. Numer. Methods Fluids, 33, pp. 55-57 (2000).

11. Hunt, B. "Asymptotic solution for dam break problem", J. Hydraul. Div, ASCE, 108, pp. 115-126 (1982).

12. Penney, W. and Thornhill, C. "The dispersion, under gravity, of a column of fluid supported on a rigid horizontal plane", Phil. Trans. Roy. Soc. A, 244, pp. 285-311 (1952).

13. Korobkin, A. and Yilmaz, O. "Initial stages of dambreaking flow", Journal of Engineering Mathematics, 63, pp. 293-308 (2009).

14. Dutykh, D. and Mitsotakis, D. "On the relevance of the dam break problem in the context of nonlinear shallow water equations", Discrete Contin. Dyn. Syst., 13, pp. 799-818 (2010).

15. Lohrasbi, A., Dolatshahi, M. and Lavaei, A. "Hydraulic model of dam break using Navier Stokes equation with arbitrary Lagrangian-Eulerian approach", International Journal of Engineering and Technology, 8, pp. 249-253 (2015).

\section{Biographies}

Alireza Lohrasbi is $\mathrm{PhD}$ graduate in Civil Engineering, University of Tehran, Iran. His research is in CFD, numerical and analytical modeling of free surfaces. He has published several technical lectures in hydrodynamic and hydraulic modeling.

Moharram Dolatshahi Pirooz is Associate Professor in the Department of Civil Engineering, University of Tehran, Iran. He is a very active engineer in the field of marine structures. He has published several technical lectures and worked with many consultants in hydrodynamic and hydraulic modeling. He recently published two papers in the field of wave height prediction. 